\title{
Mechanism-anchored profiling derived from epigenetic networks predicts outcome in acute lymphoblastic leukemia Xinan Yang ${ }^{1}$, Yong Huang ${ }^{1}$, James L Chen ${ }^{1}$, Jianming Xie ${ }^{2}$, Xiao Sun ${ }^{2}$ and Yves A Lussier*1,3,4
}

\begin{abstract}
Address: ${ }^{1}$ Center for Biomedical Informatics and Section of Genetic Medicine, Department of Medicine, The University of Chicago, Chicago, IL 60637, USA, ${ }^{2}$ State Key Laboratory of Bioelectronics, Southeast University, 210096 Nanjing, PR China, ${ }^{3}$ The University of Chicago Cancer Research Center, and The Ludwig Center for Metastasis Research, The University of Chicago, Chicago, IL 60637, USA and ${ }^{4}$ The Institute for Genomics and Systems Biology, and the Computational Institute, The University of Chicago, Chicago, IL 60637, USA
\end{abstract}

E-mail: Xinan Yang - xyang2@uchicago.edu; Yong Huang - yongh@uchicago.edu; James L Chen - jlchen27@yahoo.com; Jianming Xie -xiejm@seu.edu.cn; Xiao Sun - xsun@seu.edu.cn; Yves A Lussier* - lussier@uchicago.edu ${ }^{*}$ Corresponding author

from 2009 AMIA Summit on Translational Bioinformatics San Francisco, CA, USA 15-17 March 2009

Published: 17 September 2009

BMC Bioinformatics 2009, I0(Suppl 9):S6 doi: 10.1186/147I-2105-10-S9-S6

This article is available from: http://www.biomedcentral.com//47I-2/05//0/S9/S6

(C) 2009 Yang et al; licensee BioMed Central Ltd.

This is an open access article distributed under the terms of the Creative Commons Attribution License (http://creativecommons.org/licenses/by/2.0), which permits unrestricted use, distribution, and reproduction in any medium, provided the original work is properly cited.

\begin{abstract}
Background: Current outcome predictors based on "molecular profiling" rely on gene lists selected without consideration for their molecular mechanisms. This study was designed to demonstrate that we could learn about genes related to a specific mechanism and further use this knowledge to predict outcome in patients - a paradigm shift towards accurate "mechanismanchored profiling". We propose a novel algorithm, PGnet, which predicts a tripartite mechanismanchored network associated to epigenetic regulation consisting of phenotypes, genes and mechanisms. Genes termed as GEMs in this network meet all of the following criteria: (i) they are co-expressed with genes known to be involved in the biological mechanism of interest, (ii) they are also differentially expressed between distinct phenotypes relevant to the study, and (iii) as a biomodule, genes correlate with both the mechanism and the phenotype.
\end{abstract}

Results: This proof-of-concept study, which focuses on epigenetic mechanisms, was conducted in a well-studied set of 132 acute lymphoblastic leukemia (ALL) microarrays annotated with nine distinct phenotypes and three measures of response to therapy. We used established parametric and non parametric statistics to derive the PGnet tripartite network that consisted of 10 phenotypes and 33 significant clusters of GEMs comprising 535 distinct genes. The significance of PGnet was estimated from empirical p-values, and a robust subnetwork derived from ALL outcome data was produced by repeated random sampling. The evaluation of derived robust network to predict outcome (relapse of ALL) was significant $(p=3 \%)$, using one hundred three-fold crossvalidations and the shrunken centroids classifier.

Conclusion: To our knowledge, this is the first method predicting co-expression networks of genes associated with epigenetic mechanisms and to demonstrate its inherent capability to predict 
therapeutic outcome. This PGnet approach can be applied to any regulatory mechanisms including transcriptional or microRNA regulation in order to derive predictive molecular profiles that are mechanistically anchored. The implementation of PGnet in R is freely available at http://Lussierlab. org/publication/PGnet.

\section{Background}

By design, predictors of outcome based on gene expression profiles are based on gene lists that do not require knowledge of biological processes or molecular mechanisms [1]. Though expression arrays have been widely studied to improve prediction of clinical outcome and to aid the decision of treatment strategy for cancer, the resulting long list of genes lacking mechanistic background is thus difficult to interpret to infer their biological or clinical implications. Additionally, a poor outcome may be caused by a diversity of molecular disorders, for which the individual contribution may vary in different patients suffering from the same cancer [2]. In some cases, profiles are accompanied with followon enrichment studies or curated annotations that predict their possible mechanisms; while in other cases, functional clustering has been proposed to understand microarray data profiles [3]. In this manuscript, we propose a novel computational strategy based on genes associated to known biological mechanisms to derive mechanism-anchored expression profiles $a b$ initio that can accurately predict disease outcome.

We hypothesized that those co-expression modules, which are predictive of outcome, can be computationally derived from genes known to regulate or to be regulated by epigenetic mechanisms in previous studies and from novel microarray expression specifically designed for a new phenotype for which the epigenetic mechanisms may not be well understood [4,5]. Nearly every cancer consists of genetic mutations of the transformed cells as well as epigenetic abnormalities of non-mutational changes to DNA that lead to alterations in gene expression [6]. While genetic abnormalities found in cancer typically affect cancerpromoting oncogenes and tumor suppressor genes, the epigenetic regulation of molecular functions involves reversible interactions which can affect gene expression such as (i) DNA methylation [7], (ii) histone modification [8], (iii) RNA transcription and the resulting proteins $[9,10]$ or miRNAs [11], that influence chromatin structure. For example, histone deacetylation and the methylation of the promoter region can affect binding of transcriptional factors to these DNA regions and result in transcriptional silencing partly due to chromatin remodeling [12]. Indeed, combination therapy with inhibitors of DNA methyltransferase and histone deacetylase is under investigation in cancer [13-15]. Additionally, epigenetic events occur in the coordinated behavior of epigenetic proteins that regulate gene expressions [15]. To demonstrate the applicability of the proposed phenotype-genotype-network" method (PGnet), a set of known biological mechanism-anchored genes are required as the "seed" (input). Although this method can be generalized to other molecular mechanisms and other diseases, we focused this study on epigenetic alterations in acute lymphoblastic leukemia (ALL).

To compute co-expression modules of genes in disease and to infer their interplay generally require the integration of data from a wide variety of sources [16,17]. For example, some computational methods have been developed to indentify shared regulatory inputs, functional pathways and genetic interactions [2,18-21]. We have also previously shown that co-expression patterns of genes found in expression arrays designed around specific phenotypes can be recapitulated in gene-phenotype relationships derived from database/literature mining [22]. Further, genome-scale reverse engineering of regulatory mechanisms in expression arrays have been developed and successfully applied in mammalian cells [23]. For example, A method called ARACNE has been shown to be effective in practice by using a mutual information theoretic approach which focuses on direct co-expression of genes $[24,25]$. However, unsupervised combinations of every molecular element that may interact via one or more intermediaries can lead to a problem of multiplicity due to the escalating number of comparisons and thus to a loss of statistical power. Another method, FunNet, addresses multiplicity by combining gene expression data with Gene Ontology [26,27] or KEGG [28] annotations and further performs transcriptional functional analysis over co-expression [29]. Another method, StAM, identifies expression signature by focusing on biological processes which can characterize subgroup of patients [2]. However, these methods are not designed to compute regulatory networks that would also be differentially expressed in multiple phenotypic contexts as well as co-expressed in each individual. Our approach differs from these previous methods in that genes are integrated to the profile signature if: i) they are associated $a b$ initio to the biological mechanism of interest (here epigenetics), and ii) they are derived from the a non-parametric statistic taking into account comprehensive expression patterns of the every gene in the microarray rather than from a subset of the differential-expressed ones. 
We hypothesize that using supervised pair-wise measurements from microarray data together with robust feature selection technology [1], we are more likely to construct meaningful, epigenetic mechanism-anchored, co-expression networks that are predictive of leukemia outcome. To this end, we propose a novel supervised nonparametric algorithm (PGnet) that builds a tripartite network derived from (i) microarray expression profiles, and (ii) prior knowledge about biological mechanisms. PGnet is designed to identify sets of mechanismanchored genes that are both consistently co-expressed across arrays and differentially expressed between phenotypic conditions.

\section{Methods}

\section{Arrays and phenotypes}

We selected a large array dataset published by the Downing research group (ALL arrays) [30] that comprises well characterized subtypes of ALL and other clinical phenotypes, including cytogenetic characteristics, molecular status and patient outcomes. The details for leukemia phenotypes and sample size are provided in Suppl. Methods (Additional file 1).

\section{Epigenetic Seed Genes - ESGs (Suppl. Methods} (Additional file I) and Suppl. Table I (Additional file 2)) Gene Ontology terms and PubMed were used to identify genes with epigenetic effects. Genes were subsequently mapped to Affymetrix probe-sets and curated into eight categories.

\section{Array analyses}

The 132 ALL arrays were normalized with the variance stabilization and calibration normalization (vsn) method [31] using Bioconductor [32,33] package compdiagTools [34]. We then applied an additional inter quartile range (IQR) filter [35] to eliminate genes lacking sufficient variation across samples in expression (Suppl. Methods (Additional file 1)).

\section{Building a phenotype - gene network (PGnet)}

The construction of a network comprising "Leukemia Phenotypes" (LPs), "epigenetic seed Genes" (ESGs) and co-expressed genes required six steps (see Figure 1 and Suppl. Methods (Additional file 1) for details on steps and equations).

Step Ia: Vector of genes co-expressed with the mechanism seed genes

All genes that co-expressed with ESGs were sorted in a vector based on the Pearson correlation coefficient (PCC). Results were denoted as $V_{E S G_{i}}, \quad i=1,2, \ldots 48$.

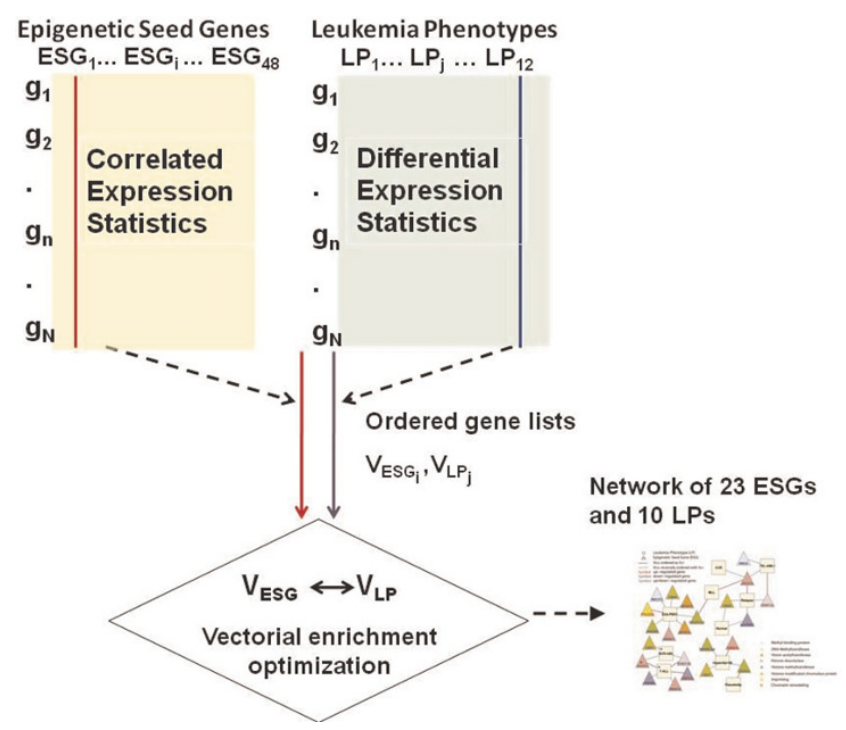

Figure I

Design of PGnet methods. Two sets of data are required to construct the network: (i) $V_{E S G}$ lists are produced by analyzing the known epigenetic "seed genes" using the pairwise standard Pearson correlation coefficient of the vsn normalized gene expression levels between ESG and all genes $g_{n}$ that meet the IQR filter criteria across all samples where genes $g_{n}(n=I, \ldots, N)$, (ii) $V_{L P}$ lists are derived by analyzing the gene's differential expression in each phenotype of interest and evaluating its significance with an adjusted ttest. The rows in both $V_{E S G}$ and $V_{L P}$ lists represent every genes in the microarray that meet the IQR filters, whereas the columns are either epigenetic seed genes in the $V_{E S G}$ lists or phenotypes in the $V_{L P}$ lists. We then use the PGnet methodology to develop a similarity vector between the epigenetic seed gene and a phenotype. We calculated the vectorial similarity between each pair of ordered expression of gene lists using a previously algorithm that we published, orderedlist (Suppl. Methods (Additional file I)) [37]. The result is a ranked list of genes that are significantly associated based on their respective $V_{E S G i}$ and $V_{L P j}$. We build a genephenotype network where relationships are similarity scores (Fig. 2). Legend: g: gene with microarray expression; ESG: epigenetic seed gene; LP: leukemia phenotype.

Step Ib: Vector of differentially expressed genes in ALL

At the same time, all genes were also sorted based on their adjusted Student t-test conducted between the phenotype of interest against the remaining pooled phenotypes. Results were denoted as $V_{L P_{j}}, \quad j=1,2, \ldots 12$. Bioconductor [32,33] package stats was used to calculate the PCC and the package Twilight [36] was used to calculate the adjusted t-score parameter.

Step 2

To compare two ordered lists of gene expressions, we used OrderedList [37-39] from Bioconductor [32,33], a 
non-parametric quantitative vectorial enrichment method that we previously published, and has been shown more sensitive to detect significant departure from a predicted distribution than semi-quantitative enrichment approaches such as the Fisher's Exact Test or the the Chi-square test. We calculated a matrix of similarity scores $M_{s}=\left(s_{i, j}\right)$, where each score $s_{i, j}$ assessed the pair-wise similarities between two vectors. The fist vector is the ordered coexpression coefficients $V_{E S G i}$ and the second one is the ordered differential expression statistics $V_{L P]}$. The similarity score gives higher weights to ranking extremes: the top and bottom ranks in both lists. In our method, we compared the ranking of genes in the co-expression set with those gene ranks from the phenotypic set. This resulted in a total of two comparisons for each phenotype/"seed gene" combination (correlation and anti-correlation).

\section{Step 3: Vectorial Enrichment Optimization (VEO)}

To evaluate the statistical significance of the similarity score, we generated 2,000 controls through the permutation of each list of gene ranks and calculated empirical p-values based on random scores. Two networks were generated. In this proof-of-concept study, the arbitrary but uniform significance threshold $(T=200$, Suppl. Methods (Additional file 1)) of included ranks was chosen to define a set of GEMs with higher differential expression in VEO that would yield a small network (Suppl. Table 2 (Additional file 3) and Figure 2), where the co-expressed with known "epigenetic seed genes" and phenotype-specific genes are the genes within the top 200 or bottom 200 in either of the two lists. An optimal length for these ordered gene lists can also be determined by unbiased optimization methods and can generate a larger network (Suppl. Table 3 (Additional file 4)), however for the purpose of simplicity of presentation - we kept the list at $\mathrm{T}=200$ for the main figures, which is within the range of length considered biologically significant in our unbiased and more comprehensive studies (Suppl. Table 3 (Additional file $4)$ ). In both the arbitrary threshold $(T=200)$ and the optimal threshold cases, the significance of PGnet were estimated by an empirical p-value of similarity scores by permutation the ranks (number of permutations $=1000$, Suppl. Methods). And the adjustment threshold of significance for vectorial similarity was conducted by controlling the false discovery rates $(\mathrm{q}$-value $=0.02)$ [40,41] (Suppl. Methods (Additional file 1)).

\section{Step 4}

For each significant seed gene/phenotype pair we considered these to be "linked." By aggregating these seed gene/phenotype pairs, we developed a tripartite network PGnet.

\section{Step 5: Visualization of the Tripartite Network}

Meaning of shapes and colors in the network: triangle (epigenetic seed genes), circle (predicted GEMs) and box (phenotypes); red (up-regulated), blue (down-regulated) and grey for vertex of a gene had more than one linkage and was up-regulated in one condition but downregulated in a different condition (Suppl. Methods (Additional file 1)). By color-coding the edges of the graph, we are providing a direction to each similarity vector, magenta line for correlation whereas turquoise for anti-correlation. With these vectors, one can judge how these genes express in a specific condition.

\section{Step 6: Biological meaning of the network}

Using Gene ontology, we conducted an enrichment of the molecular function and biological processes among the genes identified in the PGnet biomodules in order to characterize biologically the network and we also reviewed the literature for the genes involved in the biomodules associated to BCR-ABL, T-ALL and hyperdiploidy. Thus the resulting set of genes termed as Genes significantly Expressed with the Mechanism (GEMs) in the epigenetic context of this network meet all of the following criteria: (i) they are co-expressed with genes known to be involved in the biological mechanism of interest, (ii) they are also differentially expressed between distinct phenotypes relevant to the study, and (iii) as a biomodule, genes correlate with both the mechanism and the phenotype.

\section{Robust predictive network and evaluation}

The predictive capabilities of the derived network were evaluated with two approaches: (a) quantitative computational studies of the accuracy of the predictor of outcome, and (b) qualitative comparison of the PGnet method to that of another reverse engineering one.

(a) To demonstrate the accuracy of the derived network to predict relapse, we performed a conservative evaluation consisting of one hundred three-fold cross-validation (CV) studies of the PGnet method. In other words, as shown in Figure 2c, the network was derived from 2/3 of the randomly selected patients and the evaluation was conducted on the remaining third. The random selection was conducted to conserve the respective group sizes (normal, cancer) and was considered a more adequate and severe control [42]. This procedure was repeated one hundred times on different random resamplings. Two different predictive methods were used as well: (i) Prediction Analysis for Microarrays Class Prediction [43] (PAM) that does not involve any machine learning and (ii) the Support Vector Machine [44] (SVM) (Suppl. Methods (Additional file 1) and Suppl. Fig. 2 (Additional file 5)). The resulting receiver operating characteristic 

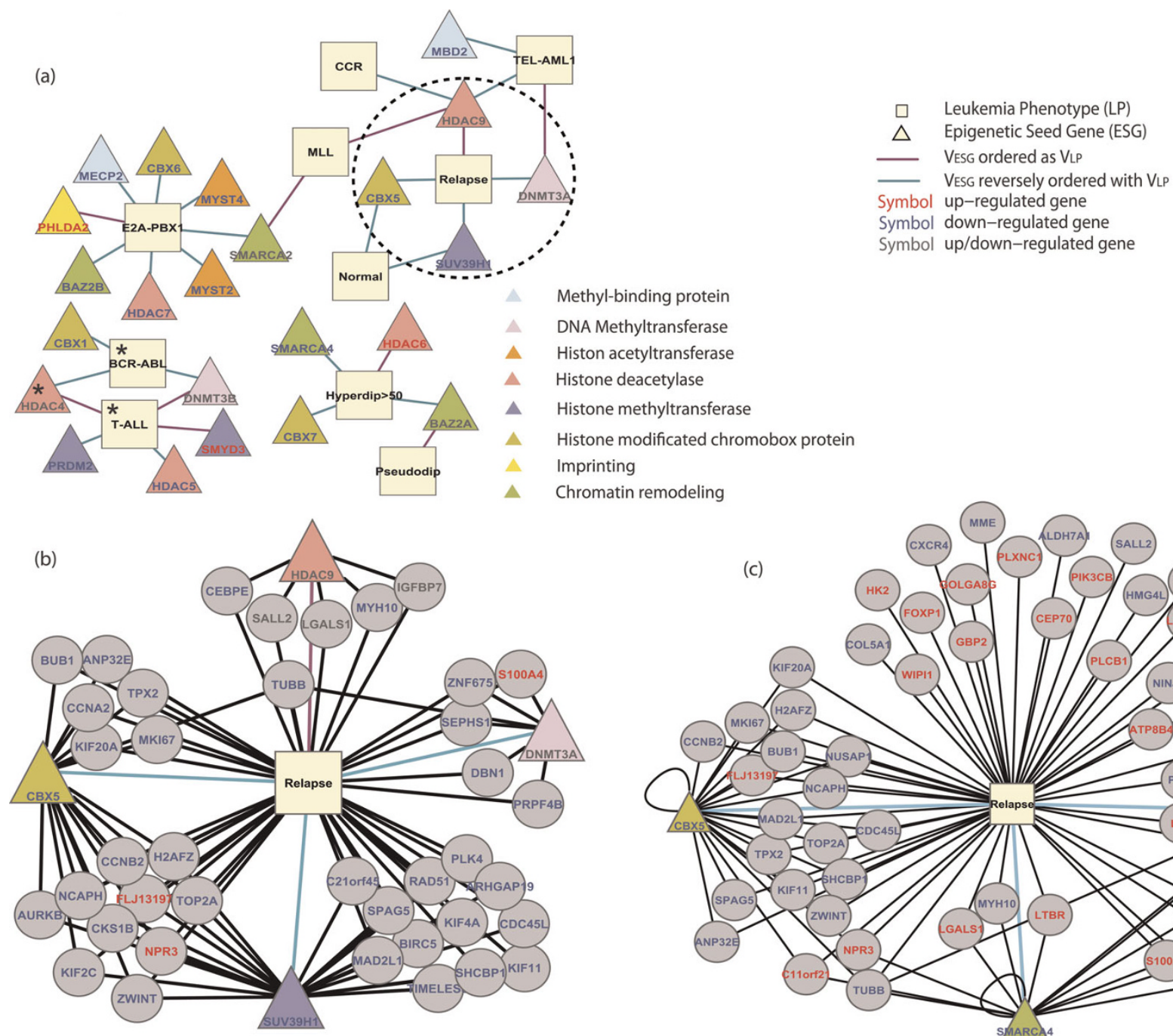

Methyl-binding protein

DNA Methyltransferase Histon acetyltransferase tone deacetylase istone methyltransferase

istone modificated chromobox protein mprinting

Chromatin remodeling

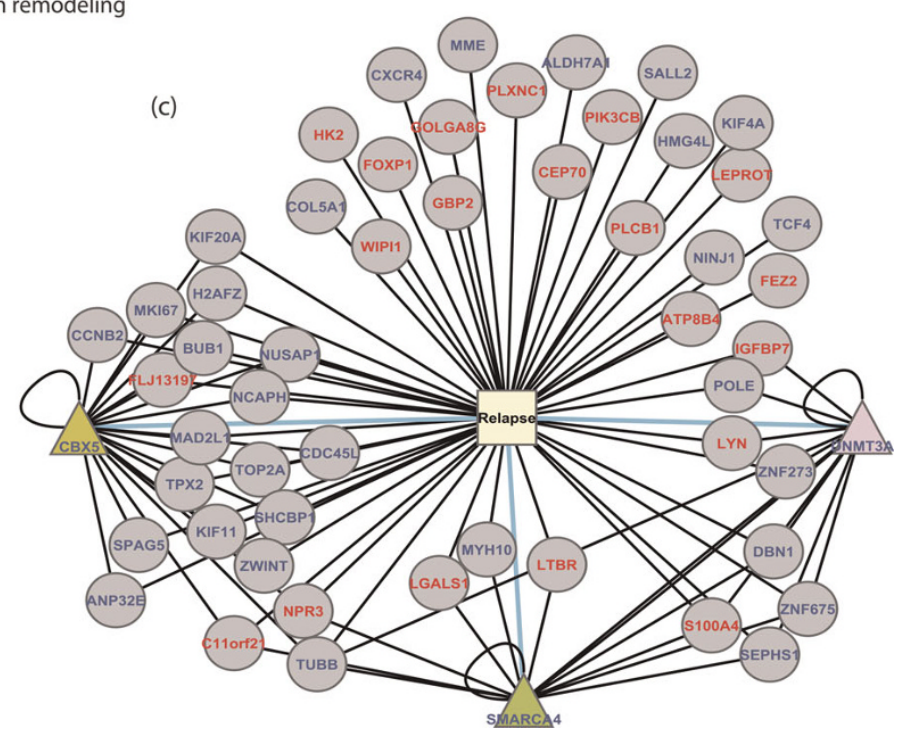

Figure 2

Network modelling of epigenetic genes - phenotypes in ALL derived from PGnet. The complete tripartite network produced by PGNet is available as Suppl. Fig. I (Additional file 14) and Suppl. Table 2 (Additional file 3). Here, panel (a) shows a bipartite subset of 33 statistically significant associations linking 23 distinct epigenetic seed genes (ESGs, yellow triangle) to 10 distinct leukemia phenotypes (yellow squares). Distinct background colors of ESG gene names indicate distinct molecular function or biological process related to or targeted by epigenetic regulation (Suppl. Table I (Additional file 2)). Two LPs and one ESG with asterisk were selected to show the details of a biomodule derived by PGnet (Figure 4). Red genes are those for which the expression is up-regulated in the associated ALL phenotype, while blue ones are with down-regulated expression, and grey ones are related to more than one phenotype with alternate up-down regulations (details of the full network in Suppl. Table 2 (Additional file 3)). Panel (b) shows a tripartite network that includes GEMs (grey circles) and focuses on the circled subset of Panel a: the "ALL relapse". Panel (c) is a robust sub-network associated to ALL outcome (relapse vs. continuous complete remission (CCR)). Three ESGs and 53 GEMs were obtained by 100 repetitions of 3-fold cross-validation of PGnet operating on the subset of ALL arrays comprising 87 patients experiencing either "CCR" or "relapse" ( $\mathrm{>}$ 32, details in Suppl. Methods (Additional file I)). Note that ALL subtypes associated PGnet in panel a) and b) were derived from all I 32 patients (in this case the biomodules of the sub-network $2 \mathrm{~b}$ pertain to patients with relapse and not everything else). The robust sub-network in panel $\mathrm{c}$ ) was conducted for training a predictor of outcome. Only 87 out of the 132 samples contained outcome information related to ALL relapse ("relapse" or "CCR" of ALL). Please note that some patients did have an outcome of secondary AML, a distinct form of leukemia, and were excluded from panel $2 c$ because this disease occurs at a later stage and the authors of the dataset did not disclose the outcome of ALL for these patients. Thus the biomodules of Figure $2 c$ overlap partially with those of Figure $2 b$. 
(ROC) curve, area under the curve (AUC) and corresponding p-values were calculated by the Bioconductor $[32,33]$ package verification [45]. A robust molecular signature is one that repeatedly appears by random sampling [1]. We further identified the GEMs correlated with the ESGs that were identified as robust [1]. The robust ESGs refer to those GEMs that were among the top $5 \%$ frequencies in the one hundred iterations of the 3fold cross-validation (Figure B in Suppl. Methods (Additional file 1)). Figure 3 illustrates the sub-network associated to the comparison between "Relapse" and the "continuous complete remission - CCR" phenotypes.

(b) Finally we compared our results to those obtained by a straightforward reverse engineering method (ARACNE).

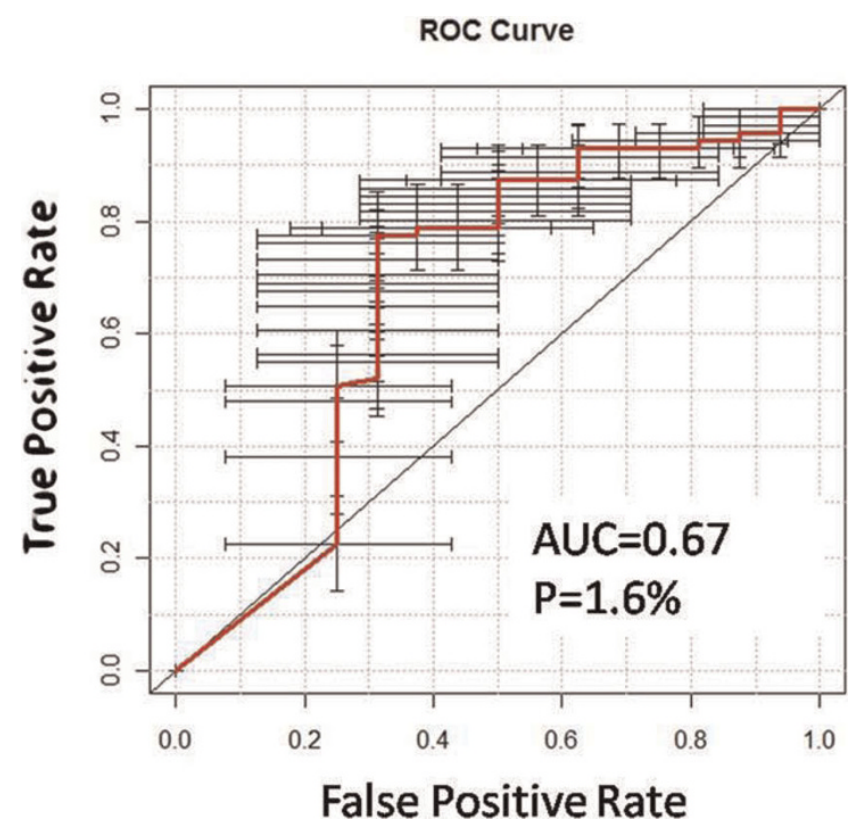

Figure 3

Evaluation of the outcome predictor of "ALL Relapse" derived from the mechanism-anchored expression profile obtained by PGnet. The 87 leukemia patients with "CCR" or "relapse" information were randomly divided into three folds, two of which were used to identify the predictor of outcome ("CCR" vs. "relapse", Figure 2c). The predictor consisted of GEMs and ESGs associated to "relapse" to train a linear SVM model, and the remaining one was used as a blinded test set. Three-fold cross-validations were repeated 100 times. The resulting Receiver Operating Characteristic (ROC) curve, the area under the curve (AUC) and corresponding $\mathrm{P}$-values were calculated by Bioconductor package verification. Horizontal and vertical "error bars" represent the $95 \%$ confidence intervals of the predictor. Regions where the error bars are above the diagonal line represent a better prediction than chance. Overall, the AUC was significantly different than that of a random predictor of "ALL relapse" ( $\mathrm{P}=1.6 \%)$.

\section{Results}

Seventy-one distinct epigenetic seed genes were identified in the literature review and denoted as "seed gene" candidates for input in PGnet (Suppl. Table 1 (Additional file 2)). In the 132 ALL arrays, 7,256 out of 12,997 unique genes and 48 out of the 71 subset of ESGs satisfy the IQR filter (bolded genes in Suppl. Table 1 (Additional file 2)). The 48 ESGs are thus enriched ab initio among genes differentially expressed in ALL samples suggesting a biological relevance in ALL ( $\mathrm{p}=5 \%$, Fisher's Exact Test).

We built a gene-phenotype network specific for epigenetic genes clusters in ALL as shown in Figures 2a and $2 b$. The derived network comprises 33 significant nodes, including eight clinical subtypes of ALL and two outcome conditions (LP, vertex in yellow box), 23 epigenetic seed genes (ESG, yellow triangle) and 535 genes that co-express with ESGs (GEM, grey circle). Three of these ESGs and 299 GEMs are up-regulated in association to their phenotype(s) as compared with their expression associated to the remaining pooled phenotypes, while 14 ESGs and 203 GEMs are downregulated. In addition, 6 ESGs and 33 GEMs were upregulated in one phenotype but down-regulated in a different phenotype, which are phenotype specifically differential expressions (see Step 3 of Methods). A summary of predictions is provided in Suppl. Table 2 (Additional file 3). These 23 ESGs genes are highly coexpressed with epigenetic genes and also highly differentially expressed in distinct ALL phenotypes groups (CBX1, CBX5, CBX6, CBX7, PHLDA2, BAZ2A, BAZ2B, MYST2, MYST4, MECP2, SMARCA2, HDAC4, HDAC5, HDAC6, HDAC7A, HDAC9, SMARCA4, SMYD3, SUV39H1, DNMT3A, DNMT3B, PRDM2 and MBD2).

To validate the prognostic ability of the genes in PGnet associated with leukemia relapse, we performed one hundred three-fold cross-validations in two ways (Suppl. Methods (Additional file 1) and Suppl. Fig. 2 (Additional file 5)). Using the PAM classification that does not require machine-learning, the predictions were accurate (AUC = $0.65, \mathrm{p}=3 \%$, Suppl. Fig. 3 (Additional file 6)). We also conducted a severe control by randomly selecting genes differentially expressed in the array and the p-values of the derived predictors ranged from $12 \%$ to $67 \%$, further corroborating that the epigenetic network derived by PGnet is associated to the relapse outcome. Using SVM machine-learning to improve the predictions in a 3-fold cross over design, PGnet achieved a AUC $=0.67(p=1.6 \%)$ (Figure 3 in this manuscript). Precision and recall of the predictor in cross-validation studies are also significant (Suppl. Fig. 4 (Additional file 7)). The evaluation confirmed the detection of co-expression biomodules 


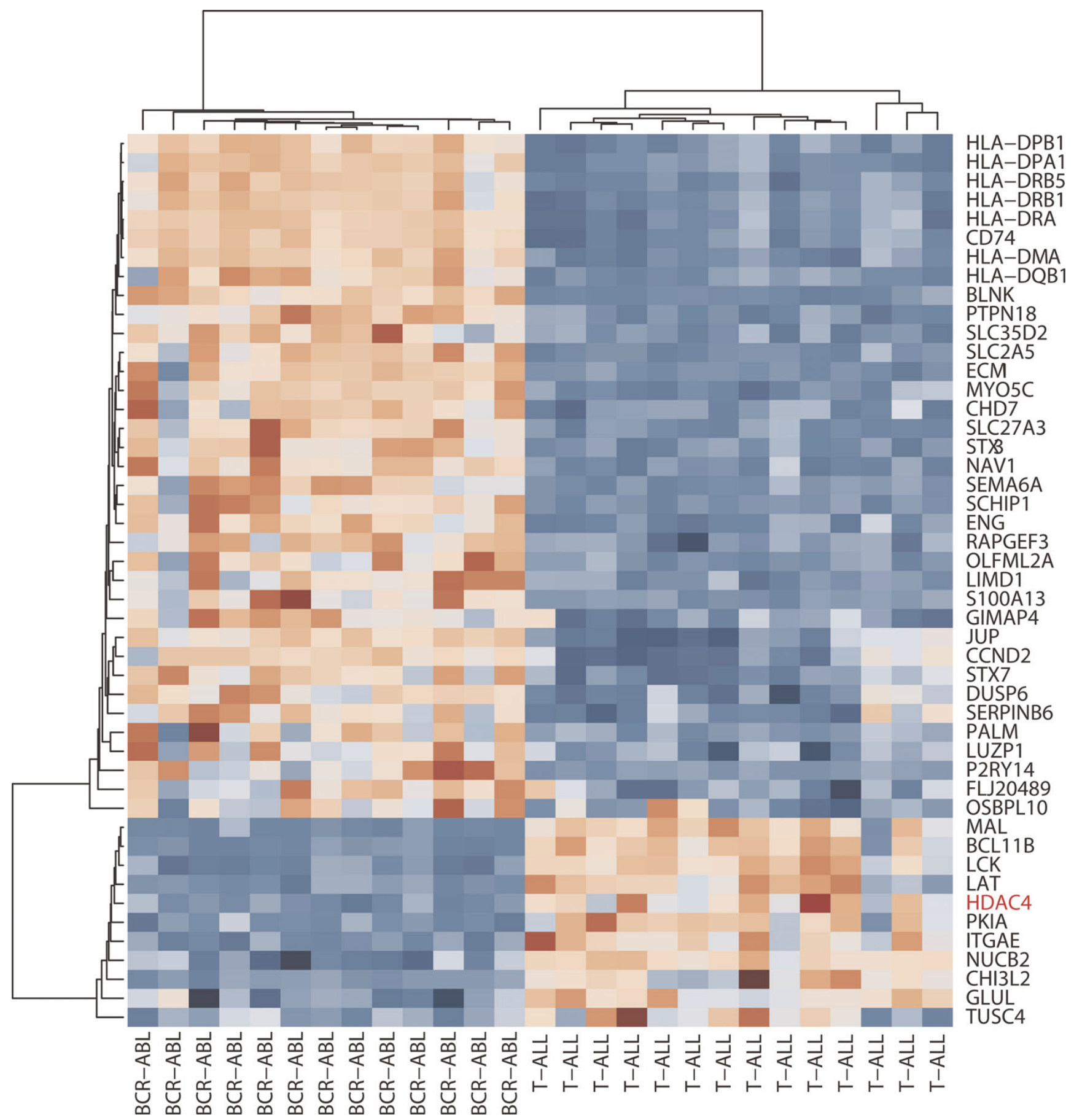

Figure 4

Details on the expression biomodule associated with BCR-ABL and T-ALL phenotypes and the epigenetic mechanism of HDAC4. The ESG (gene symbol in red) is positively correlated with part of its GEMs $(n=10)$ as downregulated in "BCR-ABL" and up-regulated in "T-ALL", and negatively correlated with another parts of its GEMs $(n=36)$. 47 genes were derived from PGnet under optimal threshold (Suppl. Table 3 (Additional file 4)). The standard full agglomerative hierarchical clustering was performed on Spearman's rank correlation distance of normalized expression levels. The resulting heatmap was drawn using Bioconductor package made4. 
associated to epigenetic alterations can be utilized in the identification of ALL with poor prognosis [46]. Supplementary Table 5 (Additional file 8) reports 52 robust GEMs together with 3 robust ESGs (CBX5, SMARCA4 and DNMT3A) associated with leukemia relapse (Suppl. Methods (Additional file 1)).

We further proceeded to identify biological enrichment in the distinct sets of genes associated with response to therapy and long-term maintenance of disease remission. There were 4 ESGs and a total of 39 GEMs associated with the phenotype "Relapse", such as CCNA2, BUB1, MAD2L1, CDC45L and CCNB2, etc, which were significantly enriched in one GO term: ATP binding (hypergeometric $\mathrm{p}=3.5 \times 10^{-6}$, Suppl. Table 4 (Additional file 9)). Interesting, ATP has been reported as treatment target of murine leukemic cells in vitro to reduce the number of leukemic clonogenic cells [47].

Of note, PGnet is designed to discover mechanismanchored biomodules that are phenotype-specific and that are consistently co-expressed across every patient. Figure 4 shows genes that are down-regulated in one phenotype and up-regulated in another phenotype and vice-versa (Suppl. Table 3 (Additional file 4)). In PGnet, 67 genes are involved in the expressional biomodules that are co-expressed with HDAC4 and DNMT3B, and yet are differentially expressed between "T-ALL" and "BCR$\mathrm{ABL}^{\prime \prime}$ phenotypes. Enrichment analysis of these genes revealed only one significant enrichment: "MHC class II receptor activity" (GO:0032395, hypergeometric p-value $=1.6 \times 10^{-11}$, Suppl. Table 4 (Additional file 9)). Further identification of the intersected regulation function of these epigenetic bio-modules requires experiments in vitro.

The GEMs derived from PGnet can distinguish their associated phenotype in addition to be co-expressed with their associated seed gene on transcript level. As an example in this study, Supplementary Figure 5 (Additional file 10) shows that the expression of 61 GEMs can clearly distinguish samples of "Hyperdiploid $>50$ " from other ALL samples. There were 4 ESGs associated with the hyperdiploid karyotypes by PGnet. HDAC6 is a class IIB histon deacetylase and identified as target of antileukemia therapy [48]. Inhibition of HDAC6 disrupts the association of $H S P 90$ with its chaperon proteins, resulting in ubiquitylation of certain oncogenes, such as Bcr$A b l$ [49]. Three other genes were also down-regulated (SMARCA4, BAZ2A and SMARCC2): SMARCA4 is a drug target candidates in hyperdiploid multiple myeloma [50]; BAZ2A is a novel nucleolar chromatin remodeling machine [51], and SMARCC2 was among the top discriminating genes in the good prognosis subgroup of MLL [52]. Moreover, GEMs identified by PGnet significantly enriched among the top-100 marker genes in previous genome-scale studies of ALL (Fisher's test $\mathrm{p}<2 \times 10^{-16}$, Suppl. Table 6 (Additional file 11))

\section{Discussion \\ Comparison of the derived network with other computational methods}

ARACNE [24,53] software was used to reverse engineer the transcriptional network in two ways. First, by providing the genes expression data for entire ALL samples as input, we compared our GEMs with genes identified by ARACNE (Result is given in Suppl. Table 7 (Additional file 12)). We provided ARACNE our 48 epigenetic seed genes and the expression data for samples in each phenotype as input, irrespectively. Subsequently, we got 12 different phenotype specific gene-gene networks. Each ARACNE network detects thousands of genes that with significant $(\mathrm{p}<0.05)$ mutual information (MI) with inputted ESGs. By design, a majority of the genes detected by ARACNE are not phenotype specific. GEMs predicted by PGnet overlap with ARACNE's prediction for 31 of 33 ESGs (Suppl. Table 8 (Additional file 13)). However, PGnet differs from ARACNE in that it provides phenotypic information left out in ARACNE.

ARACNE, FunNet and PGnet provide co-regulation networks as an output and are thus "related"; however, they differ in several important ways: (i) PGnet and FunNet combine supervised technology and non-parametric methodology while ARACNE uses information theory; (ii) inputs to PGnet are expression levels and phenotypic associations of interest such as seed genes whereas FunNet requires full expression together with a reference list of all transcripts to be analyzed and ARACNE uses expression data exclusively; (iii) FunNet abstracts transcriptional functions from co-expression layer; and consequently (iv) PGnet's output is a tripartite network consisting of co-regulated genes and clinical/ genetic characteristics of interest while ARACNE's or FunNet's outputs are uni-partite graphs. (v) The significant threshold of PGnet relates to the complete ordering of all genes to be analyzed whereas the significant threshold of FunNet is related to the coexpression of single gene. (vi) PGnet not only provides a degree of association between phenotypes but also sheds light on whether there was concordance in the directionality of the changes in expression level.

PGnet parallelizes two input vectors and finds sets of GEMs via vectorial enrichment optimization. Using measurements of differential expression and co-expression together, PGnet is more reliable in discovering phenotype-specific biomodules that are consistent across 
every patient than a simplified method that analyzes the expressed pattern of the epigenetic seed genes (ESGs) alone. First, a simplified method identifies none of GEMs from PGnet. However, we have shown some evidence indicating that the GEMs are more likely to be involved in specific epigenetic events than those directly calculated to be correlated to a phenotype of interest. Second, simpler alternate methods relying on co-expression or differential expression separately would identify only a the subset of ESGs from PGnet (data not shown), because these methods use an arbitrary threshold for significance of each gene and neglect the joint analysis of co-expression patterns with those of differential expression. In contrast, the "ESG-phenotype" linkage, which we proposed in PGnet, would be significant even if the epigenetic seed gene itself is not "significantly" differentially expressed in the linked phenotype (for instance, the seed genes that are not self-linked in Suppl. Fig. 1 (Additional file 14)).

Biologically, PGnet is an attractive technique as we know that mechanism-related genes have similar patterns of expression [4,20], and pathological mechanisms are easier to understand than genes by clinicians. Additionally, the non-parametric rank-correlation algorithm that we previously developed for Bioconductor can use the full range of the expression data for discovery instead of arbitrary statistical cut-offs [37-39]. We have extended it to derive phenotype-genotype correlations based on prior knowledge in addition to gene expression. Moreover, this tri-partite network allows to view genes for which the expression is specific to a phenotype of interest and also anchored to a biological mechanism.

\section{Future studies and limitations}

Epigenetic gene regulation is one among many possible mechanisms involved in disease-specific gene aberrant activation. Better predictors of outcome can be developed using a more comprehensive number of biological mechanisms. The PGnet method could be expanded to a broader variety of biological mechanisms in order to provide more accurate mechanism-anchored profiles that predict therapeutic outcome (e.g. transcriptional and microRNA networks [54], Gene Ontology terms, KEGG, etc), however additional methods are required to control for multiplicity of mechanism while preserving accuracy of the derived tripartite networks. In addition, this PGnet is a supervised method that relies on prior knowledge about seed genes or gene products that regulate epidemic processes. Therefore, PGnet may "skew" the network accordingly, which may reflect only subset of the real regulatory relationship. Further improvement for finding disease associated and seedgene regulated genes will likely require a refined assessment of co-expression, e.g. mutual information [24,55], instead of linear Pearson coefficient [37]. By design, PGnet identifies biomodules that are consistently co-expressed with the mechanism seed genes across all patient samples. However, there could exist mechanisms that are only co-expressed in some specific phenotypes and otherwise the co-expression patterns are lost. These particular biomodules may also contribute to mechanism-anchored predictors and require further methodological developments for their ascertainment. Future evaluations comparing the PGnet-derived predictors in other datasets are required, and we intend to proceed with multi-mechanism profiling that would in theory achieve higher precision and recall.

\section{Conclusion}

We introduced and evaluated a novel algorithm, PGnet, to identify mechanism-anchored co-expression networks and to predict therapeutic outcome. PGnet differs from previous reverse engineering methods in that it provides a more comprehensive output consisting of a tripartite network of expression similarity between genes, biological mechanisms and clinical phenotypes. Additionally, statistical significance is conducted over expression ordering inclusive of the complete array.

Trained on epigenetic mechanisms, PGnet accurately classified patients in the leukemia subtype and the relapse group, and these results suggest that a more comprehensive multi mechanism-based profile may achieve higher accuracy scores. The proposed method is scalable, in principle, to other mechanisms such as transcriptional networks, microRNA-regulated or Gene Ontology classes. In addition, the produced "similarity linkages" between mechanisms and genes comprise magnitude and direction (correlated or anti-correlated), which could also be utilize to infer regulation (activation or suppression) [15].

\section{Competing interests}

The authors declare that they have no competing interests.

\section{Authors' contributions}

Lussier was responsible of the overall experimental design and contributed to the R\&D of the methods. Yang contributed significantly to the design, methods development and implementation, as well as the majority of the computations. Xie and Sun contributed to the design, methods and epigenetic gene lists. Yang, Huang, Chen, and Lussier contributed to the evaluation, discussion and conclusion. 


\section{Additional material}

\section{Additional file 1}

Supplementary Methods - Supplementary Methods.

Click here for file

[http://www.biomedcentral.com/content/supplementary/14712105-10-S9-S6-S1.doc]

\section{Additional file 2}

Supplementary Table 1 - The eight categories of epigenetic key terms and their corresponding 71 epigenetic seed genes.

Click here for file

[http://www.biomedcentral.com/content/supplementary/14712105-10-S9-S6-S2.doc]

\section{Additional file 3}

Supplementary Table 2 - Thirty-three significant "LP-ESG" linkages contributed by corresponding GEMs (parameter $T=200$ )

Click here for file

[http://www.biomedcentral.com/content/supplementary/14712105-10-S9-S6-S3.doc]

\section{Additional file 4}

Supplementary Table 3 - The predicted GEMs of 117 significant "LPESG" linkages based on optimal parameters (Ts)

Click here for file

[http://www.biomedcentral.com/content/supplementary/14712105-10-S9-S6-S4.doc]

\section{Additional file 5}

Supplementary Figure 2 - The Design of the two Evaluations (A and B). Click here for file

[http://www.biomedcentral.com/content/supplementary/14712105-10-S9-S6-S5.doc]

\section{Additional file 6}

Supplementary Figure 3 - The ROC curve of the computational evaluation method B of PGnet-predicted GEMs and ESGs signature associated with ALL relapse.

Click here for file

[http://www.biomedcentral.com/content/supplementary/14712105-10-S9-S6-S6.doc]

\section{Additional file 7}

Supplementary Figure 4 - The precision_recall results of the computational evaluation A of PGnet-predicted GEMs and ESGs signature associated with ALL relapse.

Click here for file

[http://www.biomedcentral.com/content/supplementary/14712105-10-S9-S6-S7.doc]

\section{Additional file 8}

Supplementary Table 5 - Robust GEMs and ESGs Predictive of Leukemia Relapse.

Click here for file

[http://www.biomedcentral.com/content/supplementary/14712105-10-S9-S6-S8.doc]

\section{Additional file 9}

Supplementary Table 4 - The significantly enriched GO items among the predicted GEMs that linking to phenotypes of interested.

Click here for file

[http://www.biomedcentral.com/content/supplementary/14712105-10-S9-S6-S9.doc]

\section{Additional file 10}

Supplementary file Figure 5 - Expression pattern of ALL phenotype "Hyperdiploid>50" specific GEMs by PGnet.

Click here for file

[http://www.biomedcentral.com/content/supplementary/14712105-10-S9-S6-S10.doc]

\section{Additional file 11}

Supplementary Table 6 - Previously reported facts about predicted GEMs.

Click here for file

[http://www.biomedcentral.com/content/supplementary/14712105-10-S9-S6-S11.doc]

\section{Additional file 12}

Supplementary Table 7 - Comparison GEMs in PGnet with genes identified by ARACNE.

Click here for file

[http://www.biomedcentral.com/content/supplementary/14712105-10-S9-S6-S12.doc]

\section{Additional file 13}

Supplementary Table 8 - Comparison of the results from ARACNE and PGnet for leukemia phenotypes.

Click here for file

[http://www.biomedcentral.com/content/supplementary/14712105-10-S9-S6-S13.doc]

\section{Additional file 14}

Supplementary Figure 1 - PGnet: significant "phenotypes in ALL GEMs - epigenetic seed genes"

Click here for file

[http://www.biomedcentral.com/content/supplementary/14712105-10-S9-S6-S14.doc]

\section{Acknowledgements}

This work was supported in part by the The Cancer Research Foundation, the NIH National Center for Multiscale Analyses of Genomic and Cellular Networks (MAGNET) IU54CAI21852, and the Natural Science Foundation of China 60771024, 60121101 and. We thank Dr. Richard Sheuermann for his contribution to the interpretation of the network. We also thank Dr. Zuhong Lu for his contribution in developing the epigenetic gene list. We would also like to acknowledge Matthew Crowson for his assistance in the revision of this manuscript.

This article has been published as part of BMC Bioinformatics Volume 10 Supplement 9, 2009: Proceedings of the 2009 AMIA Summit on Translational Bioinformatics. The full contents of the supplement are available online at http://www.biomedcentral.com/I47I-2105/10?issue=S9 


\section{References}

I. Michiels S, Koscielny S and Hill C: Prediction of cancer outcome with microarrays: a multiple random validation strategy. Lancet 2005, 365(9458):488-492.

2. Lottaz C and Spang R: Molecular decomposition of complex clinical phenotypes using biologically structured analysis of microarray data. Bioinformatics 2005, 2 I(9): | 97|-1978.

3. Pan W: Incorporating gene functions as priors in modelbased clustering of microarray gene expression data. Bioinformatics 2006, 22(7):795-80I.

4. Barabasi $A L$ and Oltvai ZN: Network biology: understanding the cell's functional organization. Nat Rev Genet 2004, 5(2): I0I-II3.

5. Pujana MA, Han JD, Starita LM, Stevens KN, Tewari M, Ahn JS, Rennert G, Moreno V, Kirchhoff T and Gold B, et al: Network modeling links breast cancer susceptibility and centrosome dysfunction. Nat Genet 2007, 39(I I): |338-I349.

6. Dillon N: Gene regulation and large-scale chromatin organization in the nucleus. Chromosome Res 2006, I4(I): I I7-I26.

7. Weber $M$ and Schubeler D: Genomic patterns of DNA methylation: targets and function of an epigenetic mark. Curr Opin Cell Biol 2007, I9(3):273-280.

8. Gelato KA and Fischle W: Role of histone modifications in defining chromatin structure and function. Biol Chem 2008 , 389(4):353-363.

9. Gangaraju VK and Bartholomew B: Mechanisms of ATP dependent chromatin remodeling. Mutat Res 2007, 618(I2):3-17.

10. Lopez-Serra L and Esteller M: Proteins that bind methylated DNA and human cancer: reading the wrong words. $\mathrm{Br} / \mathrm{Cance}$ 2008, 98(I2): |88|-|885.

II. Burgess R, Jenkins $R$ and Zhang $Z$ : Epigenetic changes in gliomas. Cancer Biol Ther 2008, 7(9): | 326-I334.

12. Feinberg AP and Tycko B: The history of cancer epigenetics. Nat Rev Cancer 2004, 4(2): I43-153.

13. Gore SD: Combination therapy with DNA methyltransferase inhibitors in hematologic malignancies. Nat Clin Pract Oncol 2005, 2(Suppl I):S30-35

14. Jones PA and Baylin SB: The epigenomics of cancer. Cell 2007. I 28(4):683-692.

15. Bock $C$ and Lengauer T: Computational epigenetics. Bioinformatics 2008, 24(I): I-10.

16. Kann MG: Protein interactions and disease: computational approaches to uncover the etiology of diseases. Brief Bioinform 2007, 8(5):333-346.

17. Loscalzo J, Kohane I and Barabasi AL: Human disease classification in the postgenomic era: a complex systems approach to human pathobiology. Mol Syst Biol 2007, 3: 124.

18. van Someren EP, Wessels LF, Backer E and Reinders MJ: Genetic network modeling. Pharmacogenomics 2002, 3(4):507-525.

19. McKinney BA, Reif DM, Ritchie MD and Moore JH: Machine learning for detecting gene-gene interactions: a review. Appl Bioinformatics 2006, 5(2):77-88.

20. Oti $M$ and Brunner HG: The modular nature of genetic diseases. Clin Genet 2007, 7I(I): I-II

21. Ideker T and Sharan R: Protein networks in disease. Genome Res 2008, I 8(4):644-652.

22. Lussier YA and Liu Y: Computational approaches to phenotyping: high-throughput phenomics. Proc Am Thorac Soc 2007 4(I): I 8-25.

23. Lewin R: First success with reverse genetics. Science 1986,233 (4760): I 59-160.

24. Margolin AA, Wang K, Lim WK, Kustagi M, Nemenman I and Califano A: Reverse engineering cellular networks. Nat Protoc 2006, I(2):662-67I

25. Ma S-K: Statistical mechanics.Singapore: World Scientific; 1985.

26. Blake JA and Harris MA: The Gene Ontology (GO) project: structured vocabularies for molecular biology and their application to genome and expression analysis. Curr Protoc Bioinformatics 2002, Chapter 7(Unit 7 2):

27. Blake JA and Harris MA: The Gene Ontology (GO) project: structured vocabularies for molecular biology and their application to genome and expression analysis. Curr Protoc Bioinformatics 2008, Chapter 7(Unit 7 2):

28. Kanehisa M, Goto $S$, Kawashima $S$ and Nakaya A: The KEGG databases at GenomeNet. Nucleic Acids Res 2002, 30: 42-46.

29. Prifti E, Zucker JD, Clement $K$ and Henegar C: FunNet: an integrative tool for exploring transcriptional interactions. Bioinformatics 2008, 24(22):2636-2638.
30. Ross ME, Zhou X, Song G, Shurtleff SA, Girtman K, Williams WK, Liu HC, Mahfouz R, Raimondi SC and Lenny N, et al: Classification of pediatric acute lymphoblastic leukemia by gene expression profiling. Blood 2003, I 02(8):295I-2959.

31. Huber W, von Heydebreck A Sultmann $H$, Poustka A and Vingron $M$ : Variance stabilization applied to microarray data calibration and to the quantification of differential expression. Bioinformatics 2002, I 8(Suppl I):S96-104.

32. Gentleman RC, Carey VJ, Bates DM, Bolstad B, Dettling M, Dudoit S, Ellis B, Gautier L, Ge Y and Gentry J, et al: Bioconductor: open software development for computational biology and bioinformatics. Genome Biol 2004, 5(1 0):R80.

33. R-Development-Core-Team: R: A Language and Environment for Statistical Computing. R Foundation for Statistical Computing, 2005; Vienna, Austria 2005

34. Scheid S, Jäger J and Lottaz C: A toolbox for performing and illustrating microarray data analyses - user's guide to the $\mathbf{R}$ package compdiagTools. CompDiag Tech Rep 2005.

35. von Heydebreck A. Huber W and Gentleman R: Differential expression with the Bioconductor Project. Bioconductor Project Working Papers 2004

36. Scheid S and Spang R: twilight; a Bioconductor package for estimating the local false discovery rate. Bioinformatics 2005 2 I ( I 2):292।-2922

37. Yang X, Bentink S, Scheid S and Spang R: Similarities of ordered gene lists. I Bioinform Comput Biol 2006, 4(3):693-708.

38. Yang $X$ and Sun $X$ : Meta-analysis of several gene lists for distinct types of cancer: a simple way to reveal common prognostic markers. BMC Bioinformatics 2007, 8: I I8

39. Lottaz C, Yang X, Scheid S and Spang R: OrderedList - a bioconductor package for detecting similarity in ordered gene lists. Bioinformatics 2006, 22( I 8):23 I5-23।6.

40. Storey J: A direct approach to false discovery rates. Journal of the Royal Statistical Society, Series B 2002, 64:479-498.

4l. Storey J: The positive false discovery rate: $A$ Bayesian interpretation and the q-value. Annals of Statistics 2003 , 3।:2013-2035.

42. Barnett V: Sample Survey Principles and Method (The second edition). Hodder Arnold; |99|.

43. Tibshirani R, Hastie T, Narasimhan B and Chu G: Diagnosis of multiple cancer types by shrunken centroids of gene expression. Proc Natl Acad Sci USA 2002, 99( ( 0):6567-6572.

44. Meye $D$, Leisch $F$ and Hornik $K$ : The support vector machine under test. Neurocomputing 2003, 55(I-2): |69-I86.

45. Mason SJ and Graham NE: Areas beneath the relative operating characteristics (ROC) and relative operating levels (ROL) curves: Statistical significance and interpretation. $Q\rfloor R$ Meteorol Soc 2002, I 28:2|45-2166.

46. Yang H, Kadia T, Xiao L, Bueso-Ramos CE, Hoshino K, Thomas DA O'Brien S, Jabbour E, Pierce S and Rosner GL, et al: Residual DNA methylation at remission is prognostic in adult Philadelphia chromosome-negative acute lymphocytic leukemia. Blood 2009, I I3(9): 1892-1898.

47. Hatta $Y$, Itoh $T$, Baba M, Miyajima $T$, Shimojima $H$, Sawada $U$ and Horie T: Purging in autologous hematopoietic stem cell transplantation using adenosine triphosphate (ATP) and 4hydroperoxycyclophosphamide (4-HC). Leuk Res 2002, 26(5):477-482.

48. Rao R, Fiskus W, Yang Y, Lee P, Joshi R, Fernandez P, Mandawat A, Atadja P, Bradner JE and Bhalla K: HDAC6 inhibition enhances I7-AAG - mediated abrogation of hsp90 chaperone function in human leukemia cells. Blood 2008, I I 2(5): I886-I893.

49. Bali P, Pranpat M, Bradner J, Balasis M, Fiskus W, Guo F, Rocha K, Kumaraswamy $S$, Boyapalle $S$ and Atadja $P$, et al: Inhibition of histone deacetylase 6 acetylates and disrupts the chaperone function of heat shock protein 90: a novel basis for antileukemia activity of histone deacetylase inhibitors. J Biol Chem 2005, 280(29):26729-26734.

50. Carrasco DR, Tonon G, Huang $Y$, Zhang $Y$, Sinha R, Feng B, Stewart JP, Zhan F, Khatry D and Protopopova M, et al: Highresolution genomic profiles define distinct clinico-pathogenetic subgroups of multiple myeloma patients. Cancer Cell 2006, 9(4):313-325.

51. Strohner R, Nemeth A, Jansa P, Hofmann-Rohrer U, Santoro R, Langst G and Grummt I: NoRC - a novel member of mammalian ISWI-containing chromatin remodeling machines. Embo J 2001, 20(I7):4892-4900.

52. Tsutsumi S, Taketani T, Nishimura K, Ge X, Taki T, Sugita K, Ishii $E$, Hanada R, Ohki $M$ and Aburatani $H$, et al: Two distinct gene expression signatures in pediatric acute lymphoblastic 
leukemia with MLL rearrangements. Cancer Res 2003, 63(16): 4882-4887.

53. Basso K, Margolin AA, Stolovitzky G, Klein U, Dalla-Favera R and Califano $A$ : Reverse engineering of regulatory networks in human B cells. Nat Genet 2005, 37(4):382-390.

54. Gennarino VA, Sardiello M, Avellino R, Meola N, Maselli V, Anand S, Cutillo L, Ballabio A and Banfi S: MicroRNA target prediction by expression analysis of host genes. Genome Res 2008.

55. Margolin AA, Nemenman I, Basso K, Wiggins C, Stolovitzky G, Dalla Favera $R$ and Califano A: ARACNE: an algorithm for the reconstruction of gene regulatory networks in a mammalian cellular context. BMC Bioinformatics 2006, 7(SuppI I):S7.

Publish with BioMed Central and every scientist can read your work free of charge

"BioMed Central will be the most significant development for disseminating the results of biomedical research in our lifetime. "

Sir Paul Nurse, Cancer Research UK

Your research papers will be:

- available free of charge to the entire biomedical community

- peer reviewed and published immediately upon acceptance

- cited in PubMed and archived on PubMed Central

- yours - you keep the copyright
BioMedcentral 